\title{
Characteristics and Mortality Risk Factors of Influenza-Associated Encephalopathy/Encephalitis in Children in a Tertiary Pediatric Hospital in China, 2016-2019
}

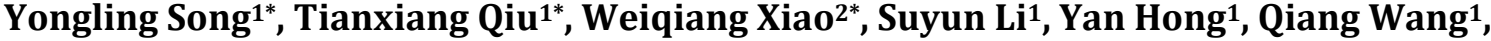 Qinglian Chen ${ }^{1}$, Xiaowei Fan1, Peiqing Li" ${ }^{1 \#}$}

${ }^{1}$ Department of Pediatric Emergency, Guangzhou Women and Children's Medical Center, Guangzhou Medical University, Guangzhou, China

${ }^{2}$ Medical Imaging Department, Guangzhou Women and Children's Medical Center, Guangzhou Medical University, Guangzhou, China

Email: \#annie_129@126.com

How to cite this paper: Song, Y.L., Qiu, T.X., Xiao, W.Q., Li, S.Y., Hong, Y., Wang, Q., Chen, Q.L., Fan, X.W. and Li, P.Q. (2021) Characteristics and Mortality Risk Factors of Influenza-Associated Encephalopathy/Encephalitis in Children in a Tertiary Pediatric Hospital in China, 2016-2019. Journal of Behavioral and Brain Science, 11, 157-170.

https://doi.org/10.4236/jbbs.2021.117012

Received: May 28, 2021

Accepted: July 17, 2021

Published: July 20, 2021

Copyright $\odot 2021$ by author(s) and Scientific Research Publishing Inc. This work is licensed under the Creative Commons Attribution International License (CC BY 4.0).

http://creativecommons.org/licenses/by/4.0/

\begin{abstract}
Background: Seasonal influenza associated neurological complications had high mortality and morbidity rates in children. In this study, we aimed to investigate the clinical characteristics and mortality risk factors in children with influenza-associated encephalopathy. Methods: Retrospectively analyze the clinical data, laboratory tests, and imaging examinations of 68 children diagnosed with influenza-associated encephalopathy from January 2016 to December 2019 at Guangzhou Women and Children's Medical Center, and the cases were divided into survival and non-survival groups by disease outcome and analyzed between two groups. Chi-square test or Mann-Whitney rank sum test was used for comparison between groups, and multivariate Logistic regression analysis was used for the analysis of risk factors for death. Results: Among the 68 children with influenza-associated encephalopathy, 40 were male, and 28 were female, aged from 3 months to 13 years, of which $66.18 \%$ $(45 / 68)$ were under 5 years old. Pathogenetic tests showed that influenza virus type A accounted for $63.24 \%$ (43/68), and influenza virus type B accounted for $36.76 \%$ (25/68). Typical brain MRI changes in childhood influenza-associated encephalopathy were bilateral symmetrical lesions of the thalamus, basal ganglia, brainstem, and cerebellum. 68 patients had a mortality
\end{abstract}

\footnotetext{
*Yongling Song, Tianxiang Qiu and Weiqiang Xiao contributed equally to this work.

${ }^{\sharp}$ Corresponding author.
} 
rate of $20.59 \%(14 / 68)$, with a significantly higher proportion of fever peak > $39^{\circ} \mathrm{C}$, Acute Disturbance of Consciousness (ADOC), and cardiac arrest in the non-survival group than in the survival group $(\mathrm{P}<0.05)$. Laboratory tests showed significantly higher in Alanine Aminotransferase (ALT), Aspartate Transaminase (AST), Creatinine Kinase (CK), Lactate Dehydrogenase (LDH), lactate, and C-Reactive Protein (CRP). And CSF protein levels in the non-survival group compared with the survivor $(\mathrm{P}<0.05)$, among them, elevated ALT, AST, LDH, and CSF protein were independent high-risk factors for death from influenza-associated encephalopathy. Conclusions: Children under 5 years of age with influenza are prone to combine neurological complications and have a higher mortality rate. Significant elevations in ALT, AST, LDH, and CSF proteins predict death from influenza-associated encephalopathy in children.

\section{Keywords}

Children, Influenza, Encephalopathy, Encephalitis, Clinical Characteristics, Risk Factor

\section{Introduction}

Influenza, belonging to the family Orthomyxoviridae, primarily affects the respiratory system and represents one of the most frequent causes of acute upper respiratory tract infections during the winter season [1] [2]. Although the infection is usually self-limiting, children have a higher risk of complications [3].

Neurological complication, often defined as Influenza-Associated Encephalitis/Encephalopathy (IAE), is rare but an important complication of influenza infection with approximatively three-quarters of cases regarding children and leading up to $30 \%$ of mortality in children [1] [4] [5]. The manifestations are heterogeneous, from simple febrile seizures to severe acute necrotizing encephalopathy. Neurological symptoms of brain injury usually appear on the same day or several days after the onset of cold symptoms, with convulsions and changes in consciousness of the most common. The common types of nervous system injury caused by influenza are Influenza-Associated Encephalopathy (IAE) [5], Reye's Syndrome, Guillain Barré Syndrome, Hemorrhagic Shock Encephalopathy Syndrome [6], and Acute Necrotizing Encephalopathy (ANE) [5] [7], among which ANE is the most serious.

For including a variety of acute encephalopathy syndromes and lacking available biomarkers, it is difficult to diagnose or predict the outcome of IAE, leading to delays for the early diagnosis of critical cases, resulting in poor prognosis and mortality. Neurological signs/symptoms caused by influenza include mild altered mental status, vertigo, epileptic status, meningitis, and demyelinating disease [6] [7] [8] [9]. Some neuroradiological features, in particular extensive changes in Magnetic Resonance Imaging (MRI), are reported to be related to disease severity. Age less than 5 years, history with comorbid disease was found 
to be the risk factors for severe IAE [8]. At present, there are few studies of mortality risk factors in large samples of children with IAE, and only single case reports or small case series on this issue are available.

Therefore, in this study, we analyzed the mortality risk factors of IAE in children admitted to Guangzhou Women and Children's Medical Center in 2016-2019. Through the early identification of high-risk factors, pediatricians could make timely interventions to promote prognosis.

\section{Methods}

\subsection{Patients}

This study included 68 patients with IAE who were admitted to Guangzhou Women and Children's Medical Center between January 2016 and December 2019. All patients were nasal and pharyngeal swab specimen positive for influenza virus A or B in by real-time reverse transcriptase-polymerase chain reaction (RT-PCR). The ethics committee approved this study of Guangzhou Women and Children's Medical Center, Guangzhou Medical University (Sui, Fuer, Kelun [2019], NO 38201). Individual written informed consent was obtained from the patients' parents or guardians.

The inclusion criteria were: 1 ) $<14$ years of age; 2 ) positive real-time PCR detection of influenza virus in nasal and pharyngeal swabs; and 3) presence of symptoms and signs of nervous system injury, such as seizures, rapid cognitive impairment, ADOC or coma.

The exclusion criteria were: 1) co-infection with other infections such as herpes simplex virus, cytomegalovirus, or bacteria; 2) with serious comorbidities such as immune deficiency, metabolic disorders, trauma, cerebrovascular disease, or brain tumor; 3) congenital neurological malformation or syndrome; or 4) any inherited condition affecting the neurological functions.

\subsection{Definition of Neurological Complications}

The definition of IAE includes the following criteria [10] [11] [12]: sudden onset of symptoms of influenza, and respiratory specimen positive for an influenza virus; the emergence or development of a neurological symptom in influenza infection, such as convulsion, alteration of consciousness, rapidly progressive coma; the abnormal neuroradiological finding in Neuroimaging, including edema, hemorrhage, and bilateral thalamic lesions, as well as electroencephalography showing a diffuse slowing of the background activity; exclusion of infection from other pathogens or non-infectious diseases such as metabolic disorders, trauma, cerebrovascular disorders, and/or brain tumors.

\subsection{Data Collection}

We take the retrospective method in this study. Data on demographics, clinical presentation, laboratory findings, microbiologic and neuroimaging findings, treatment, response to therapy, and outcome were collected. Demographic data 
included age, gender, and onset month. Clinical data included fever (peak temperature and time of fever lasting), respiratory symptoms, headache, vomiting, and neurological symptoms (convulsion, ADOC, rapidly progressive coma).

We routinely took these tests when patients admitted to the hospital on day 1 . Results of routine blood test, the Comprehensive Metabolic Panel (CMP) (including Alanine Transaminase (ALT), Aspartate Transaminase (AST), Creatine Kinase (CK), Lactate Dehydrogenase (LDH), C-Reactive Protein (CRP), and lactate), routine and biochemical test in CSF, and pathogenetic test in blood/nasal swabs/CSF were collected on admission day aslaboratory and microbiologic data. And neuroimaging data included CT and MRI, diagnosed by a senior pediatric radiologist (more than ten years experiences), and were reviewed again for IAE.

For influenza virus testing, Real-time Reverse Transcription Polymerase Chain Reaction (rRT-PCR) was performed using the influenza virus A/B dual fluorescence quantitative RT-PCR kit (Guangdong Guanyin Pharmaceutical Technology Co. LTD, China). The influenza virus A subtype identification was performed using the real-time RT-PCR kit (Dan Gene Co., Ltd, Sun Yat-Sen University, China).

For clinical routine biochemical testing, an automatic analyzer (Automatic Analyzer 7600, Hitachi High-Technologies Corporation) was used.

The specimens of plasma and cerebrospinal fluid (CSF) were tested in the included cases. The equipment included: a microplate reader (Thermo Fisher Scientific, Multiscan MK3) and a fluorescence analyzer (Perkin Elmer, Victor X5). The reagents used were Cyt-C ELISA Kit (Cusabio, Wuhan, China, No: CSB-E08530h), Apo-ONE ${ }^{\oplus}$ Homogeneous Caspase-3/7 Assay (Promega, USA, No: G7790), Amplex ${ }^{\oplus}$ Red Hydrogen Peroxide/Peroxidase Assay Kit (Invitrogen, USA, No: A22188), AA (PDGF-AA) ELISA Kit (Cusabio, Wuhan, China, No: CSBE17143h), VEGF ELISA Kit (MultiSciences, Hangzhou, China, No: 70-EK1832), Human aquaporin-4 antibody (AQP-4 Ab) ELISA Kit (Cusabio, Wuhan, China, No: CSB-E13568h), Human antiNMDAR Antibody ELISA Kit (Fine Test, Zurich, Switzerland, No: EH4166), Human MOG (Myelin-oligodendrocyte glycoprotein) ELISA Kit (Fine Test, Zurich, Switzerland, No: EH0896), and Lipid Peroxidation MDA Assay Kit (Beyotime Biotechnology Co., Ltd., Shanghai, China, No: S0131).

\subsection{Data Analyses}

All statistical analyses were performed using the SPSS 22.0 software (IBM Corp., Armonk, NY, USA). Categorical data were presented as frequency with the corresponding percentage, and continuous data were presented as median with the Interquartile Range (IQR). The Chi-squared or Fisher exact test was used to compare categorical variables. To determine the independent contribution of each factor to the case outcomes, multiple logistic regression analysis was performed. Two-tailed P-values of $<0.05$ were considered statistically significant. 


\section{Results}

\subsection{Demographics and Clinical Characteristics}

There was a total of 68 patients with IAE in this study, 40 males and 28 females, aged 3 months to 13 years, of whom $66.18 \%$ (45/68) were younger than 5 years old, were not vaccinated against influenza and had no underlying disease. The patients' main clinical manifestations at the time of admission were fever, convulsions, Acute Disturbance of Consciousness (ADOC), and vomiting. IAE occurred mainly in January (7 cases), February (8 cases), March (11 cases), June ( 9 cases), and December (13 cases). The patients were divided into survival and non-survival groups according to clinical outcome. We observed $20.5 \%$ patients (14 cases) dead in this research, of which the proportion of children with temperature $>39^{\circ} \mathrm{C}$, ADOC and cardiac arrest was significantly higher in the nonsurvival group ( $100 \%$ vs. $51.85 \% \mathrm{P}<0.05,100 \%$ vs. $40.7 \% \mathrm{P}<0.001,1.85 \%$ vs. $35.7 \% \mathrm{P}<0.05$ ) (see Table 1 ) and the proportion of children requiring tracheal intubation with ventilator-assisted ventilation was significantly higher in the non-survival group compared to the survival group $(35.7 \%$ vs. $1.85 \% \mathrm{P}<0.05)$.

\subsection{Laboratory Findings}

In this study, the median blood while cell (WBC) count in laboratory tests was $8.55(2.4-29.6) \times 10^{9} / \mathrm{L}$. In all cases, there were $39.71 \%$ patients $(27 / 68)$ with elevated Alanine Aminotransferase (ALT), 52.94\% patients (36/68) with elevated Aspartate Aminotransferase (AST), 38.24\% patients (26/68) with elevated Creatine Kinase (CK), 58.82\% patients (40/68) with elevated lactate dehydrogenase (LDH), and both $53.45 \%$ patients (31/68) with elevated C-Reactive Protein (CRP) or lactate. Comparing to the survival group, there was a statistically significant elevation of ALT, AST, CK, LDH, CRP, and LDH in the non-survival group ( $\mathrm{P}<$ $0.05)$, as shown in Table 1 . The median CSF protein in the non-survival group and survival group was respectively $1.90 \mathrm{~g} / \mathrm{L}(0.12-34.5)$ and $0.34 \mathrm{~g} / \mathrm{L}(0.12$ 12.4). The difference between the two groups was statistically significant $(\mathrm{P}<$ $0.05)$.

\subsection{Neuroimaging Findings}

In this study, except for 4 patients who failed to complete neuroimaging examination due to their unstable condition, the other 64 cases underwent brain CT or MRI. The typical brain MRI in children with IAE could be found in some patients, presenting symmetric high signal in the bilateral thalamus, basal ganglia, medulla oblongata, pontocerebellar and cerebellum as shown in Figure 1. But the other showed various and non-specific manifestations, which may show scattered abnormal signal shadows in the cerebral hemispheres, cerebral edema, and some did not show apparent abnormalities (see Figure 2).

\subsection{Treatment}

After admission, the patients were routinely monitored and given temperature 
Table 1. Demographics and clinical characteristics of the children with IAE.

\begin{tabular}{|c|c|c|c|c|}
\hline & $\begin{array}{c}\text { Total } \mathrm{n}=68 \text { Number } \\
(\% / \mathrm{IQR})\end{array}$ & $\begin{array}{c}\text { Survivors } \mathrm{n}=54 \\
\text { Number }(\% / \mathrm{IQR})\end{array}$ & $\begin{array}{c}\text { Non-survivors } \mathrm{n}=14 \\
\text { Number }(\% / \mathrm{IQR})\end{array}$ & P-value \\
\hline \multicolumn{5}{|l|}{ Gender } \\
\hline Male & $40(58.82)$ & $33(61.11)$ & $7(50)$ & 0.5468 \\
\hline \multicolumn{5}{|l|}{ Age } \\
\hline$\leq 5$ years old & $45(66.18)$ & $37(68.52)$ & $8(57.14)$ & 0.5288 \\
\hline \multicolumn{5}{|l|}{ Clinical manifestations } \\
\hline Fever $>39^{\circ} \mathrm{C}$ & $42(61.76)$ & $28(51.85)$ & $14(100)$ & 0.0005 \\
\hline time of fever lasting (days) & $3(1-22)$ & $4(1-22)$ & $3(1-17)$ & 0.43 \\
\hline Respiratory symptoms & $30(44.12)$ & $23(42.59)$ & $7(50)$ & 0.5586 \\
\hline Convulsion & $48(33.30)$ & $36(30.30)$ & $12(36.70)$ & \\
\hline Less than 3 times & $24(64.71)$ & $18(66.67)$ & $6(57.14)$ & \multirow{2}{*}{0.5415} \\
\hline More than 3 times & $24(35.29)$ & $18(33.33)$ & $6(42.86)$ & \\
\hline Headache & $12(17.65)$ & $11(20.37)$ & $1(7.14)$ & 0.4351 \\
\hline Vomiting & $22(32.35)$ & $16(29.63)$ & $6(42.86)$ & 0.3559 \\
\hline Fatigue & $19(27.94)$ & $17(31.48)$ & $2(14.29)$ & 0.3186 \\
\hline $\mathrm{ADOC}$ & $36(52.90)$ & $22(40.7)$ & $14(100)$ & $<0.0001$ \\
\hline Cardiac arrest & $6(8.82 \%)$ & $1(1.85)$ & $5(35.7)$ & 0.001 \\
\hline \multicolumn{5}{|l|}{ Routine blood test } \\
\hline Leukocyte $\left(\times 10^{9} / \mathrm{L}\right)$ & $8.55(2.4-29.6)$ & $8.5(2.4-29.6)$ & $8.8(3.2-21.7)$ & 0.8837 \\
\hline Lymphocytes $\left(\times 10^{9} / \mathrm{L}\right)$ & $1.89(0.32-16.01)$ & $2.03(0.32-16.01)$ & $1.55(0.38-5.1)$ & 0.4105 \\
\hline Monocyte $\left(\times 10^{9} / \mathrm{L}\right)$ & $0.58(0.06-3.45)$ & $0.6(0.15-3.45)$ & $0.38(0.06-0.97)$ & 0.08 \\
\hline \multicolumn{5}{|l|}{ Comprehensive metabolic panel } \\
\hline \multicolumn{5}{|l|}{ Serum } \\
\hline $\operatorname{ALT}(\mathrm{U} / \mathrm{L})$ & $33(9-7304)$ & $28.5(9-1885)$ & $70(13-7304)$ & 0.0074 \\
\hline AST (U/L) & $69(19-16,770)$ & $51.5(19-1681)$ & $176(43-16,770)$ & $<0.0001$ \\
\hline $\mathrm{CK}(\mathrm{U} / \mathrm{L})$ & $246(28-16,549)$ & $204(28-16,549)$ & $669(86-9350)$ & 0.0069 \\
\hline $\mathrm{LDH}(\mathrm{U} / \mathrm{L})$ & $357(197-11,962)$ & $322(197-2495)$ & $870(332-11,962)$ & $<0.0001$ \\
\hline $\mathrm{CRP}(\mathrm{mg} / \mathrm{dL})$ & $5(0-141.95)$ & $3.78(0-141.95)$ & $11.35(2.43-128.06)$ & 0.037 \\
\hline Lactate (mmol/L) & $1.7(0.5-11.1)$ & $1.6(0.5-6.4)$ & $2.45(1-11.1)$ & 0.0036 \\
\hline \multicolumn{5}{|l|}{ CSF } \\
\hline Protein $(g / L)$ & $0.44(0.12-34.5)$ & $0.34(0.12-12.4)$ & $1.895(0.12-34.5)$ & $<0.0001$ \\
\hline Leukocyte $\left(\times 10^{6} / \mathrm{L}\right)$ & $6(0-350)$ & $6(0-350)$ & $9(1-28)$ & 0.919 \\
\hline \multicolumn{5}{|l|}{ Pathogenetic test } \\
\hline FA & $43(63.2)$ & $35(64.8)$ & $8(57.1)$ & \multirow{2}{*}{0.7569} \\
\hline FB & $25(36.8)$ & $19(35.2)$ & $6(42.9)$ & \\
\hline \multicolumn{5}{|l|}{ Treatments } \\
\hline Oseltamivir & $68(100.00)$ & $54(100.00)$ & $14(100.00)$ & 1 \\
\hline Gammaglobulin & $68(100.00)$ & $54(100.00)$ & $14(100.00)$ & 1 \\
\hline Methylprednisolone & $68(100.00)$ & $54(100.00)$ & $14(100.00)$ & 1 \\
\hline Ventilator-assisted ventilation & $24(35.29)$ & $12(22.22)$ & $12(85.71)$ & $<0.0001$ \\
\hline
\end{tabular}


Table 2. Results of the multivariate analysis of the mortality risk factors in children with IAE.

\begin{tabular}{ccc}
\hline & OR (OR 95\% CI $)$ & $P$ \\
\hline ALT & $0.951(95 \%$ CI $0.920-0.982)$ & 0.002 \\
AST & $1.047(95 \%$ CI $1.017-1.077)$ & 0.002 \\
LDH & $0.994(95 \%$ CI $0.988-0.999)$ & 0.015 \\
Lactate & $1.010(95 \%$ CI $1.00-01.02)$ & 0.06 \\
CRP & $1.010(95 \%$ CI $0.99-1.02)$ & 0.356 \\
CK & $1.00(95 \%$ CI $0.999-1.01)$ & 0.47 \\
ADOC & $1.45(95 \%$ CI $0.53-0.42)$ & 0.84 \\
Fever & $1.00(95 \%$ CI $0.98-1.02)$ & 0.01 \\
CSF protein & $4.385(95 \%$ CI $1.432-13.425)$ &
\end{tabular}
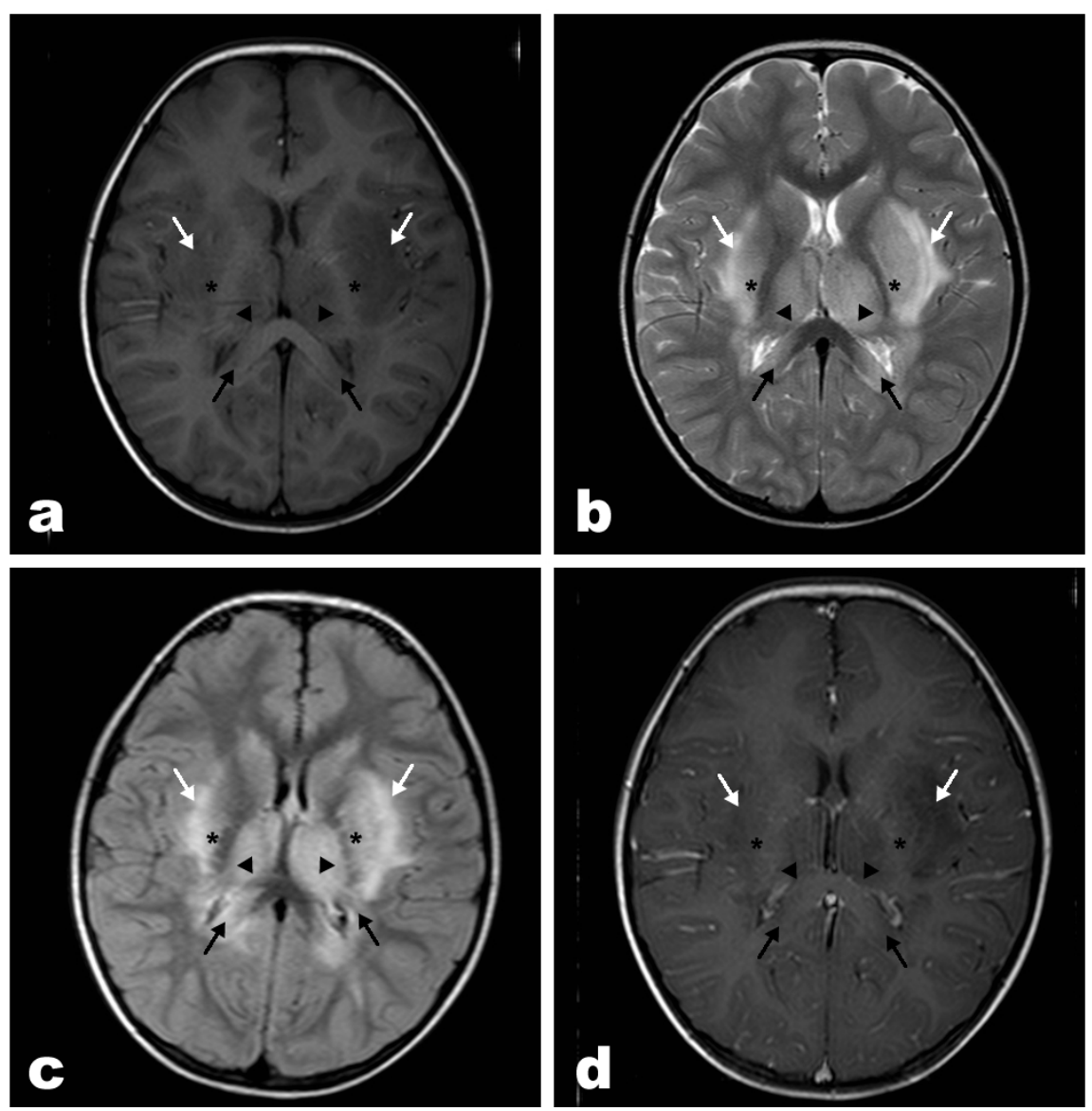

Figure 1. A 3-year-old boy with acute necrotizing encephalitis, with fever for 2 days and convulsion once. Bilateral dorsal thalamus and basal ganglia were swollen. Multiple asymmetrically patch like abnormal signal shadows were observed in bilateral dorsal thalamus $(\boldsymbol{\Delta})$, basal ganglia $\left(^{*}\right)$, external capsule (white arrow) and corpus callosum (black arrow), with blurred edges. Axial T1WI (a) showed slightly lower signal intensity, while T2WI (c) and T2WI-FLAIR (d) showed high signal intensity. Contrast-enhanced scan (d) showed no enhancement in the lesion and slightly increased shadow of small vessels on the brain surface. 

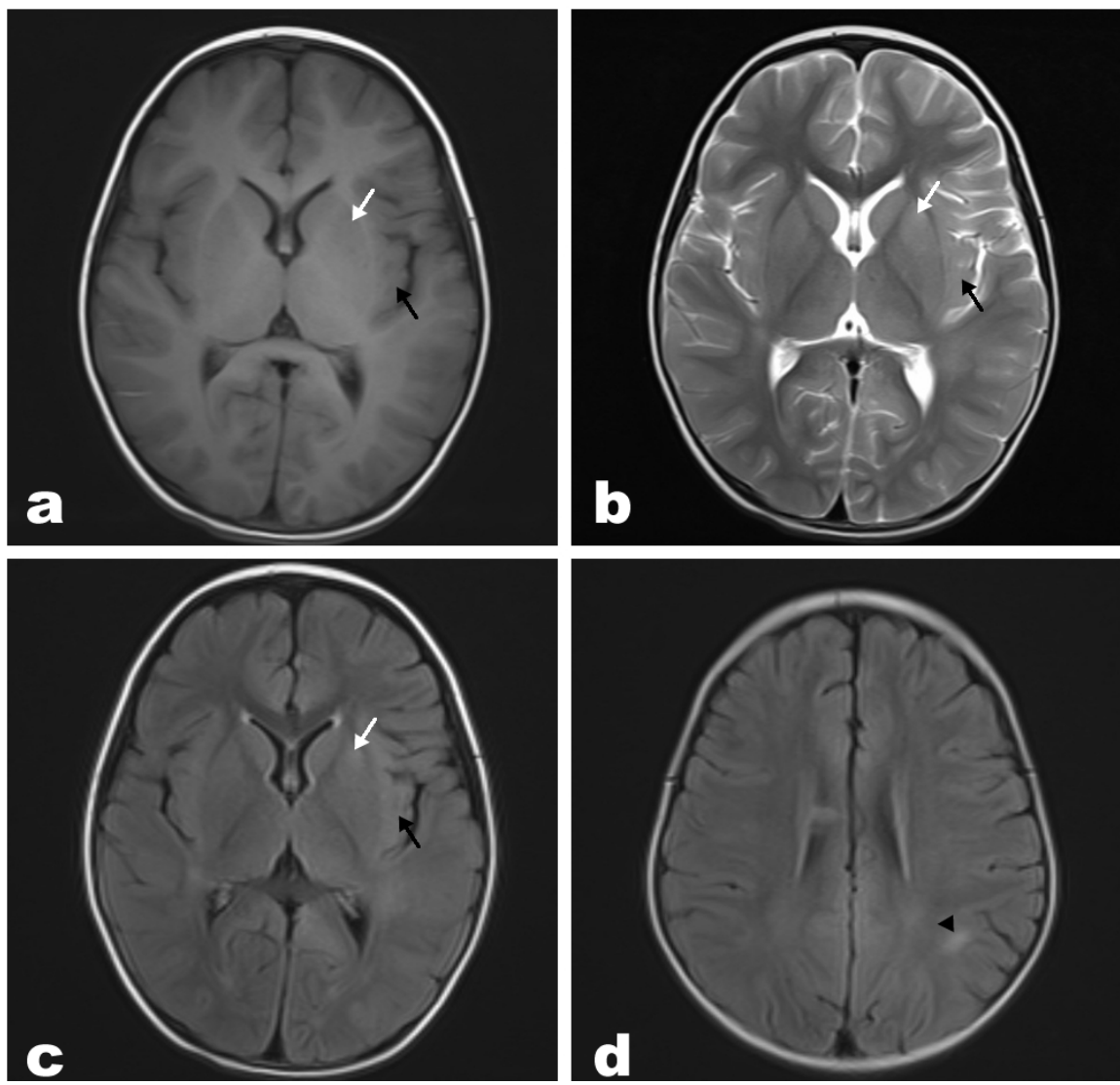

Figure 2. A 3-year-old boy with influenza-related encephalopathy, with movement disorders 5 days and fever for 3 days. Small patches of abnormal signal were observed in the left lupulate nucleus (white arrow) and left insular lobe (black arrow), with slightly lower signal on T1WI (a), slightly higher signal on T2WI (b), and slightly higher signal on T2WI-FLAIR (c). Small patches of abnormal signal $(\boldsymbol{\Delta})$ were seen in the white matter of the left parietal lobe, and T2WI-FLAIR (d) showed slightly higher signa.

reduction and anticonvulsive treatment. All patients were treated with oseltamivir, methylprednisolone $(10 \mathrm{mg} / \mathrm{kg} \cdot \mathrm{d})$, and gammaglobulin $(1 \mathrm{~g} / \mathrm{kg} \cdot \mathrm{d} \cdot 2 \mathrm{~d})$ immediately after diagnosis. Twenty-four patients were ventilated with tracheal intubation due to central respiratory failure, and were given mannitol, concentrated sodium, and nutritional support therapy. After the above treatment, 39.7\% (27/68) of patients recovered, $39.7 \%$ (27/68) developed sequelae, and 20.6\% $(14 / 68)$ died.

\subsection{Mortality Risk Factors}

The multiple logistic regression analysis showed that high levels of ALT (OR = 0.951 (95\% CI 0.920 - 0.982)), AST (OR = 1.047 (95\% CI 1.017 - 1.077)), LDH $(\mathrm{OR}=0.994(95 \% \mathrm{CI} 0.988-0.999))$, and CSF protein $(\mathrm{OR}=4.385(95 \% \mathrm{CI}$ 1.432 - 13.425)) were high risk factors for death from IAE (see Table 2).

\section{Discussion}

Influenza is a common infectious disease worldwide [13]. While mainly invad- 
ing the respiratory tract, influenza viruses can cause neurological damage in some cases, leading to a series of neurological syndromes. The morbidity and mortality rates of neurological damages due to influenza are high, especially in children [4] [14], and the mortality rate was as high as $20.5 \%$ in this study. Since the 1990s, cases of IAE have been reported in Asia, especially in Japan, and even more frequently around the world during the $2009 \mathrm{H} 1 \mathrm{~N} 1$ pandemic [15]. However, for lacking specific tests for the diagnosis of IAE, it makes the early diagnosis and treatment of the disease difficult. There are relatively few reports of IAE in children, with most single cases or small case series reported. We studied 68 patients diagnosed with IAE admitted to our hospital from 2016 to 2019, to analyze their epidemiology, clinical symptoms, laboratory and imaging tests, treatment, outcomes, and analyze the high-risk factors for fatal cases. The results indicate that children under 5 years of age with influenza are prone to combine neurological complications and have a higher mortality rate, significant elevations in ALT, AST, LDH, and CSF proteins predict death from influenza-associated encephalopathy in children.

It has been previously reported that influenza in children at a younger age or with underlying diseases is more likely to combine neurological complications, especially in children younger than 5 years of age [16] [17] [18]. In this study, $66.18 \%(45 / 68)$ of the patients were $\leq 5$ years old, which is consistent with the reports. All patients in this study were previously healthy and without underlying diseases. Therefore, it should be given close attention when the onset of neurological symptoms in influenza in healthy children. In this study, we find that the onset of IAE occurred mainly from December to March, which is consistent with the high influenza season. It is currently unknown whether influenza vaccines can prevent IAE or reduce the disease's extent. There is a report showing that patients with IAE were unvaccinated against influenza [19]. In this study, none of the 68 patients were vaccinated against influenza, suggesting the potential importance of influenza vaccine for children, and therefore vaccination of healthy preschool children in the pre-high influenza season is necessary.

Influenza-associated encephalopathy lacks specific clinical manifestations, and convulsions and impaired consciousness have been previously reported as the most common neurological symptoms of influenza-associated encephalopathy [8]. The main clinical manifestations of the cases in this study are fever, convulsions, ADOC, and respiratory symptoms could be mild or even absent. Moreover, the number of convulsions in the non-survival group did not differ from that in the survival group, indicating that the number of convulsions cannot be used to indicate disease severity.

Previous studies have reported that IAE is mainly caused by influenza virus type A, especially H1N1 [2] [20] [21]. In this study, we detected influenza virus type $A$ at $63.2 \%(43 / 68)$ in the patients and influenza virus type B at $36.8 \%$ (25/68), showing that influenza virus type $A$ is the primary infection source of IAE. However, there was no significant difference in the pathogenic distribution 
between the non-survival and survival groups, indicating that influenza virus type B may be causing relatively minor neurological damage, but leading to death in severe cases.

Some laboratory tests were found to be markedly abnormal in this study, such as reduced platelets, elevated ALT, AST, CK, LDH, and CSF proteins level. The above indicators were more abnormal in the non-survival group than the survival group, which was statistically significant. Furthermore, Logistic regression analysis showed that elevated ALT, AST, LDH, and CSF protein were independent risk factors for death in IAE, suggesting that the influenza virus could cause damage to the liver, muscle, and other tissues in addition to neurological complications. Previous studies have also reported that elevated AST, LDH, CK, and reduced platelets are associated with a poor prognosis of IAE [5] [22] [23] [24]. Therefore, patients found to have abnormalities of the above indicators in clinical practice should be given intensive care and immediate treatment to minimize death occurrence.

Brain MRI of IAE can show characteristic changes and has an important role in diagnosing and clinical staging of neurological complications of influenza. The typical brain MRI manifestations of children with IAE were previously reported symmetrical abnormalities of bilateral thalamus, basal ganglia area, medulla oblongata, and pons [20] [25] [26], which are consistent with the present study. The brain MRI of pediatric IAE is specific and contributes to the disease's early diagnosis.

The pathogenesis of IAE is still unclear, and it is generally believed that it may result from immune damage or direct viral invasion. Similar to previous studies reported [19], few patients in this study had elevated CSF leukocytes. At the same time, CSF protein was elevated in this study, and it was significantly higher in the non-survival group compared with the survival group, while logistic analysis showed elevated CSF protein as a high-risk factor for death in IAE. This suggests that neurological injury may be immune-mediated, or direct viral invasion, or vascular inflammation, rather than a simple inflammatory response. However, whether influenza viruses can invade the nervous system is controversial, and some studies have reported positive results for viral RNA in CSF and brain tissue in only a few patients [27]. As reported, all patients in this study had negative results for influenza virus RNA on CSF.

There is no standard treatment protocol for IAE, and currently used protocols include antiviral therapy, immunotherapy (pulse therapy with corticosteroids, immunoglobulins), plasma replacement and hypothermia, but it is unclear which protocol is more effective [28] [29] [30] [31]. For oseltamivir inhibiting the viral expression and reducing the inflammation, some studies report that antiviral therapy may be effective, while guidelines of Infectious Diseases Society of America recommend oseltamivir in the presence of IAE [32]. Although pulse therapy with corticosteroids and immunoglobulins remains controversial, it is currently used widely in clinical practice, for considering IAE is related to im- 
mune-mediated damage to the nervous system. All cases in this study were given oseltamivir, and pulse therapy with corticosteroids and immunoglobulins after diagnosis of influenza infection, so it was impossible to compare the difference in efficacy between the survival and non-survival groups.

Our study presents several limitations. The present study is a single-center retrospective analysis with a relatively small number of cases and a lack of long-term assessment in children, which has limitations, and more studies are needed to address these issues.

\section{Conclusion}

In conclusion, IAE should be considered when neurological symptoms are present for influenza in children under 5 years of age. Elevated ALT, AST, LDH, and CSF proteins are high-risk factors for death from IAE. Future larger studies are needed to understand the pathogenesis of IAE, explore biomarkers for assessing neurological involvement and disease severity, and follow the long-term prognosis of the disease.

\section{Authors' Contribution}

Song, Y.L., Qiu, T.X. and Xiao, W.Q. carried out the studies, participated in collecting data, and drafted the manuscript; Li, S.Y., Hong, Y. and Wang, Q. performed the statistical analysis and participated in its design; Chen, Q.L. and Fan, X.W. participated in acquisition, analysis, or interpretation of data; Li, P.Q. review revised manuscript. All authors read and approved the final manuscript.

\section{Conflicts of Interest}

The authors declare no conflicts of interest regarding the publication of this paper.

\section{References}

[1] Britton, P.N., Dale, R.C., Blyth, C.C., Macartney, K., Crawford, N.W., Marshall, H., Clark, J.E., Elliott, E.J., Webster, R.I., Cheng, A.C., et al. (2017) Influenza-Associated Encephalitis/Encephalopathy Identified by the Australian Childhood Encephalitis Study 2013-2015. The Pediatric Infectious Disease Journal, 36, 1021-1026. https://doi.org/10.1097/INF.0000000000001650

[2] Yildizdas, D., Kendirli, T., Arslankoylu, A.E., Horoz, O.O., Incecik, F., Ince, E. and Ciftci, E. (2011) Neurological Complications of Pandemic Influenza (H1N1) in Children. European Journal of Pediatrics, 170, 779-788. https://doi.org/10.1007/s00431-010-1352-y

[3] Wang, G.F., Li, W. and Li, K. (2010) Acute Encephalopathy and Encephalitis Caused by Influenza Virus Infection. Current Opinion in Neurology, 23, 305-311. https://doi.org/10.1097/WCO.0b013e328338f6c9

[4] Akins, P.T., Belko, J., Uyeki, T.M., Axelrod, Y., Lee, K.K. and Silverthorn, J. (2010) H1N1 Encephalitis with Malignant Edema and Review of Neurologic Complications from Influenza. Neurocritical Care, 13, 396-406. https://doi.org/10.1007/s12028-010-9436-0 
[5] Morishima, T., Togashi, T., Yokota, S., Okuno, Y., Miyazaki, C., Tashiro, M. and Okabe, N. (2002) Encephalitis and Encephalopathy Associated with an Influenza Epidemic in Japan. Clinical Infectious Diseases. An Official Publication of the Infectious Diseases Society of America, 35, 512-517. https://doi.org/10.1086/341407

[6] San Millan, B., Teijeira, S., Penin, C., Garcia, J.L. and Navarro, C. (2007) Acute Necrotizing Encephalopathy of Childhood: Report of a Spanish Case. Pediatric Neurology, 37, 438-441. https://doi.org/10.1016/j.pediatrneurol.2007.07.007

[7] Tabarki, B., Thabet, F., Al Shafi, S., Al Adwani, N., Chehab, M. and Al Shahwan, S. (2013) Acute Necrotizing Encephalopathy Associated with Enterovirus Infection. Brain \& Development, 35, 454-457. https://doi.org/10.1016/j.braindev.2012.07.001

[8] Paksu, M.S., Aslan, K., Kendirli, T., Akyildiz, B.N., Yener, N., Yildizdas, R.D., Davutoglu, M., Yaman, A., Isikay, S., Sensoy, G., et al. (2018) Neuroinfluenza: Evaluation of Seasonal Influenza Associated Severe Neurological Complications in Children (a Multicenter Study). Child s Nervous System, 34, 335-347.

https://doi.org/10.1007/s00381-017-3554-3

[9] Aydin, H., Ozgul, E. and Agildere, A.M. (2010) Acute Necrotizing Encephalopathy Secondary to Diphtheria, Tetanus Toxoid and Whole-Cell Pertussis Vaccination: Diffusion-Weighted Imaging and Proton MR Spectroscopy Findings. Pediatric Radiology, 40, 1281-1284. https://doi.org/10.1007/s00247-009-1498-9

[10] Sugaya, N. (2002) Influenza-Associated Encephalopathy in Japan. Seminars in Pediatric Infectious Diseases, 13, 79-84. https://doi.org/10.1053/spid.2002.122993

[11] Shiomi, M. (2011) Pathogenesis of Acute Encephalitis and Acute Encephalopathy. Nihon Rinsho Japanese Journal of Clinical Medicine, 69, 399-408.

[12] Howard, A., Uyeki, T.M. and Fergie, J. (2018) Influenza-Associated Acute Necrotizing Encephalopathy in Siblings. Journal of the Pediatric Infectious Diseases Society, 7, e172-e177. https://doi.org/10.1093/ipids/piy033

[13] Azziz Baumgartner, E., Dao, C.N., Nasreen, S., Bhuiyan, M.U., Mah, E.M.S., Al Mamun, A., Sharker, M.A., Zaman, R.U., Cheng, P.Y., Klimov, A.I., et al. (2012) Seasonality, Timing, and Climate Drivers of Influenza Activity Worldwide. The Journal of Infectious Diseases, 206, 838-846. https://doi.org/10.1093/infdis/jis467

[14] Wong, K.K., Jain, S., Blanton, L., Dhara, R., Brammer, L., Fry, A.M. and Finelli, L. (2013) Influenza-Associated Pediatric Deaths in the United States, 2004-2012. Pediatrics, 132, 796-804. https://doi.org/10.1542/peds.2013-1493

[15] Okuno, H., Yahata, Y., Tanaka-Taya, K., Arai, S., Satoh, H., Morino, S., Shimada, T., Sunagawa, T., Uyeki, T.M. and Oishi, K. (2018) Characteristics and Outcomes of Influenza-Associated Encephalopathy Cases Among Children and Adults in Japan, 2010-2015. Clinical Infectious Diseases. An Official Publication of the Infectious Diseases Society of America, 66, 1831-1837. https://doi.org/10.1093/cid/cix1126

[16] Wada, T., Morishima, T., Okumura, A., Tashiro, M., Hosoya, M., Shiomi, M. and Okuno, Y. (2009) Differences in Clinical Manifestations of Influenza-Associated Encephalopathy by Age. Microbiology and Immunology, 53, 83-88. https://doi.org/10.1111/j.1348-0421.2008.00100.x

[17] Havers, F., Fry, A., Peacock, G. and Finelli, L. (2014) Influenza Vaccination and Treatment in Children with Neurologic Disorders. Therapeutic Advances in Vaccines, 2, 95-105. https://doi.org/10.1177/2051013613519217

[18] Wilking, A.N., Elliott, E., Garcia, M.N., Murray, K.O. and Munoz, F.M. (2014) Central Nervous System Manifestations in Pediatric Patients with Influenza A H1N1 Infection during the 2009 Pandemic. Pediatric Neurology, 51, 370-376. https://doi.org/10.1016/j.pediatrneurol.2014.04.026 
[19] Kedia, S., Stroud, B., Parsons, J., Schreiner, T., Curtis, D.J., Bagdure, D., Brooks-Kayal, A.R., Glode, M.P. and Dominguez, S.R. (2011) Pediatric Neurological Complications of 2009 Pandemic Influenza A (H1N1). Archives of Neurology, 68, 455-462. https://doi.org/10.1001/archneurol.2010.318

[20] Turgut, M. and Sagiroglu, S. (2020) Suboccipital Decompressive Craniectomy in a Case of Complex Chiari Malformation Complicated with Influenza A (H1N1) Necrotizing Encephalopathy. Child s Nervous System, 36, 1335-1336. https://doi.org/10.1007/s00381-020-04659-7

[21] Teke, T., Coskun, R., Sungur, M., Guven, M., Bekci, T.T., Maden, E., Alp, E., Doganay, M., Erayman, I. and Uzun, K. (2009) H1N1 Influenza and Experience in Three Critical Care Units. International Journal of Medical Sciences, 8, 270-277. https://doi.org/10.7150/ijms.8.270

[22] Nagao, T., Morishima, T., Kimura, H., Yokota, S., Yamashita, N., Ichiyama, T., Kurihara, M., Miyazaki, C. and Okabe, N. (2008) Prognostic Factors in Influenza-Associated Encephalopathy. The Pediatric Infectious Disease Journal, 27, 384-389. https://doi.org/10.1097/INF.0b013e318162a13b

[23] Li, S., Hu, D., Li, P., Xiao, W., Li, H., Liu, G., Song, Y., Ning, S., Peng, Q., Zhao, D., et al. (2021) Parameters Indicating Development of Influenza-Associated Acute Necrotizing Encephalopathy: Experiences from a Single Center. Medical Science Monitor, 27, e930688. https://doi.org/10.12659/MSM.930688

[24] Song, Y., Li, S., Xiao, W., Shen, J., Ma, W., Wang, Q., Yang, H., Liu, G., Hong, Y., Li, P., et al. (2021) Influenza-Associated Encephalopathy and Acute Necrotizing Encephalopathy in Children: A Retrospective Single-Center Study. Medical Science Monitor, 27, e928374. https://doi.org/10.12659/MSM.928374

[25] Kimura, S., Ohtuki, N., Nezu, A., Tanaka, M. and Takeshita, S. (1998) Clinical and Radiological Variability of Influenza-Related Encephalopathy or Encephalitis. Acta Paediatrica Japonica: Overseas Edition, 40, 264-270. https://doi.org/10.1111/j.1442-200X.1998.tb01925.X

[26] Voudris, K.A., Skaardoutsou, A., Haronitis, I., Vagiakou, E.A. and Zeis, P.M. (2001) Brain MRI Findings in Influenza A-Associated Acute Necrotizing Encephalopathy of Childhood. European Journal of Paediatric Neurology: EJPN: Official Journal of the European Paediatric Neurology Society, 5, 199-202. https://doi.org/10.1053/ejpn.2000.0511

[27] Fujimoto, S., Kobayashi, M., Uemura, O., Iwasa, M., Ando, T., Katoh, T., Nakamura, C., Maki, N., Togari, H. and Wada, Y. (1998) PCR on Cerebrospinal Fluid to Show Influenza-Associated Acute Encephalopathy or Encephalitis. The Lancet, 352, 873-875. https://doi.org/10.1016/S0140-6736(98)12449-2

[28] Mizuguchi, M., Ichiyama, T., Imataka, G., Okumura, A., Goto, T., Sakuma, H., Takanashi, J.I., Murayama, K., Yamagata, T., Yamanouchi, H., et al. (2021) Guidelines for the Diagnosis and Treatment of Acute Encephalopathy in Childhood. Brain \& Development, 43, 2-31. https://doi.org/10.1016/j.braindev.2020.08.001

[29] Khandaker, G., Zurynski, Y., Buttery, J., Marshall, H., Richmond, P.C., Dale, R.C., Royle, J., Gold, M., Snelling, T., Whitehead, B., et al. (2012) Neurologic Complications of Influenza A(H1N1)pdm09: Surveillance in 6 Pediatric Hospitals. Neurology, 79, 1474-1481. https://doi.org/10.1212/WNL.0b013e31826d5ea7

[30] Mizuguchi, M., Yamanouchi, H., Ichiyama, T. and Shiomi, M. (2007) Acute Encephalopathy Associated with Influenza and Other Viral Infections. Acta Neurologica Scandinavica, 115, 45-56. https://doi.org/10.1111/j.1600-0404.2007.00809.x

[31] Kawashima, H., Togashi, T., Yamanaka, G., Nakajima, M., Nagai, M., Aritaki, K., 
Kashiwagi, Y., Takekuma, K. and Hoshika, A. (2005) Efficacy of Plasma Exchange and Methylprednisolone Pulse Therapy on Influenza-Associated Encephalopathy. Journal of Infection, 51, E53-E56. https://doi.org/10.1016/j.jinf.2004.08.017

[32] Tunkel, A.R., Glaser, C.A., Bloch, K.C., Sejvar, J.J., Marra, C.M., Roos, K.L., Hartman, B.J., Kaplan, S.L., Scheld, W.M., Whitley, R.J., et al. (2008) The Management of Encephalitis: Clinical Practice Guidelines by the Infectious Diseases Society of America. Clinical Infectious Diseases: An Official Publication of the Infectious Diseases Society of America, 47, 303-327. https://doi.org/10.1086/589747 